\title{
Multiple Carrier Frequency Offset and Channel State Estimation in the Fading Channel
}

\author{
Brad W. Zarikoff*, James K. Cavers ${ }^{\dagger}$ \\ School of Engineering Science, Faculty of Applied Sciences \\ Simon Fraser University \\ Burnaby, BC, Canada V5A 1S6 \\ $\left\{{ }^{*}\right.$ bwzariko@sfu.ca, ${ }^{\dagger}$ cavers@sfu.ca $\}$
}

\begin{abstract}
The joint estimation of multiple carrier frequency offsets (CFOs) and channel state information (CSI) in a multibase MIMO downlink system is considered here for the case of a fading channel. The multiple CFOs result from independent local oscillators at each basestation (BS), and cause accumulation of phase errors that compromise beamforming accuracy. Using previously devised multiple CFO estimation techniques for simultaneously transmitted training sequences in a non-fading channel, we begin by examining the effects of fading on a linear channel state estimate. An example illustrates that a fade rate threshold can be defined after which linear channel estimation is infeasible. Because of the dependence of the CFO estimators on the CSI estimate, this threshold applies to the CFO estimates as well. As a way to combat the channel variation, the maximum likelihood metric is modified to accommodate a parameterized fading channel estimate.
\end{abstract}

\section{INTRODUCTION}

To achieve a high-capacity multiuser MIMO system, channel state information (CSI) is the most powerful tool available to the communications engineer. With high quality CSI, the inherent diversity of the multipath in the system can be used to create virtual channels that are independent of one another. These channels can be used as a means to eliminate inter-user interference without having to resort to time and/or frequency expansion. If the channel state is static, then these virtual channels can be created once and used indefinitely; for fading channels, periodic channel estimation is necessary for update purposes. The virtual channels are very sensitive to errors in the CSI, as demonstrated in [1].

As a means to increase system capacity further, coordinated multibase MIMO (CMB-MIMO) systems, as proposed in $[2],[3],[4]$, share information in order to create a single, very large MIMO system. For CMB-MIMO, the carrier frequency offset (CFO) between each basestation (BS) is capable of disrupting the virtual channels. For example, if the downlink of a CMB-MIMO system relies on spatial zero forcing (ZF) to separate signals to different users, degraded CSI resulting from CFO can cause some loss of orthogonality, and therefore increased interference and reduced capacity [5].

For BS frequency accuracy of, for example, $\varepsilon_{0}=1 \mathrm{ppm}$, and a static or slowly time-varying channel, it is the CFOs that

This work was supported by a National Sciences and Engineering Research Council (NSERC) of Canada Post Graduate Scholarship. Submitted to the 2008 IEEE $68^{t h}$ Vehicular Technology Conference. cause the deterioration of CSI with time. To ensure that the estimation of both CFO and CSI is done in an efficient and timely manner, simultaneous training sequence transmission is considered. Estimators that can achieve the Cramer-Rao lower bound have been demonstrated [6],[7].

In the case of fast fading channels, however, the assumption that the CFO is the main cause of deterioration for the CSI no longer holds. The accuracy of joint CFO and CSI estimation in a fading channel must be studied. This paper investigates methods of simultaneous estimation of multiple CFOs and CSIs in a time-varying fading channel. It appears to be the first analysis of this problem.

\section{BACKGROUND}

\section{A. System Model}

In this study, we consider a time-varying and frequency-flat channel. An extension to the metric used here and presented in [7] accommodates channels that exhibit static frequency selective characteristics. At the receiver, the signal is downconverted to baseband and matched filtered. The output of the matched filter contains sufficient statistics at one sample per symbol, given that the influence of the CFO over the signaling pulse duration is negligible. The signal as observed at the receiver is affected by multiple CFOs; the reason for these CFOs can be attributed to either an independent Doppler effect on each multi-path [6] or to independent local oscillators (LOs) at each transmit antenna array [5].

We will assume that the multiple CFOs are a result of the independent LOs posed in the CMB-MIMO system. An orthogonal multiuser technique is used by multiple BSs to mitigate interference between transmitted streams. BS CFOs cause phase errors that accumulate in the time between mobile subscriber (MS) channel estimation and receipt of those estimates at the BSs, as well as during the transmission. The phase errors degrade the orthogonal channels that were established by the coordinated beamformers, causing increased interference and reduced capacity. This provides the motive for CFO estimation in multibase systems. Compensation will be considered in a future publication.

The proposed model is shown in Fig. 1, and consists of $B$ BSs, each with $N_{T}$ antennas. The complex scalar $h_{b, t, m}(n)$ contains the channel gain between the $t^{t h}$ antenna of the $b^{t h}$ 


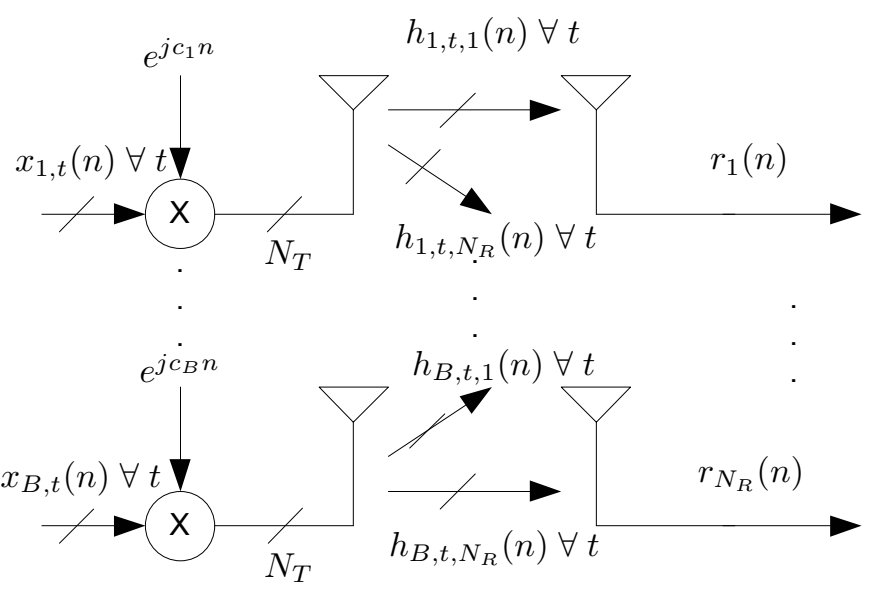

Fig. 1. Coordinated MU-MIMO downlink model

BS and the $m^{\text {th }}$ antenna at the MS, where the index $n$ refers to the $n^{t h}$ symbol interval and $N_{R}$ is the number of antennas at the MS. The CFO is considered as the frequency offset of the LO from an ideal carrier frequency. Of course, the MS has a CFO of its own, and any estimates of BS CFO are relative to the MS CFO. For the purposes of this paper, it is assumed that the MS CFO is zero. The received sample from the $m^{t h}$ receive antenna at the $n^{\text {th }}$ symbol interval is

$$
r_{m}(n)=\sum_{b=1}^{B} \sum_{t=1}^{N_{T}} x_{b, t}(n) h_{b, t, m}(n) e^{j c_{b} n}+z_{m}(n) .
$$

The scalar $c_{b}$ is the unwanted phase offset of the $b^{t h}$ BS normalized by the symbol rate (detailed in the following section), the complex scalar $x_{b, t}(n)$ contains the transmitted symbol from the $t^{t h}$ antenna at the $b^{t h} \mathrm{BS}$, and the complex scalar $z_{m}(n)$ contains the additive white Gaussian noise at the $m^{\text {th }}$ MS antenna. The $x_{b, t}(n)$ satisfy $\sum_{t}\left|x_{b, t}(n)\right|^{2}=P_{B S}$, where $P_{B S}$ and $E_{B S}=P_{B S} T_{s}$ are the total transmit power and transmit energy from each BS, respectively. The variance of the individual random channel gain $h_{b, t, m}(n)$ is set to $E\left[\left|h_{b, t, m}(n)\right|^{2}\right]=1$, and does not reflect realistic system parameters such as shadowing or any particular cellular structure. The communication system is made up of a homogenous cloud of BSs and MSs.

The CFO estimates will be made using training sequences (TSs) that have been transmitted synchronously from the $B N_{T}$ transmit antennas. The TS matrix for each transmit antenna $\mathbf{X}_{b, t}=\operatorname{diag}\left(x_{b, t}(1) \cdots x_{b, t}(N)\right)$ after CFO rotation is defined as a new matrix $\mathbf{U}$. The set of equations used to describe $\mathbf{r}$ are:

$$
\begin{aligned}
& \mathbf{r}_{m}=\mathbf{U h}_{m}+\mathbf{z}_{m} \\
& =\left[r_{m}(1) \cdots r_{m}(N)\right]^{T} \\
& \mathbf{U}=\left[\begin{array}{llll}
\mathbf{U}_{1,1} & \mathbf{U}_{1,2} & \cdots & \mathbf{U}_{B, N_{T}}
\end{array}\right] \\
& \mathbf{U}_{b, t}=\mathbf{X}_{b, t} \mathbf{D}_{b}\left(c_{b}\right) \\
& \mathbf{h}_{m}=\left[\mathbf{h}_{1,1, m}^{T} \mathbf{h}_{1,2, m}^{T} \cdots \mathbf{h}_{B, N_{T}, m}^{T}\right]^{T}
\end{aligned}
$$

$$
\begin{aligned}
\mathbf{h}_{b, t, m} & =\left[h_{b, t, m}(1) \cdots h_{b, t, m}(N)\right]^{T} \\
\mathbf{z}_{m} & =\left[z_{m}(1) \cdots z_{m}(N)\right]^{T},
\end{aligned}
$$

where $\mathbf{D}_{b}\left(c_{b}\right)$ is the $N \times N$ BS CFO matrix detailed in Section II-B. For conciseness, the vector $\mathbf{r}$ is used to represent the concatenation of the $N_{R} \mathbf{r}_{m}$ vectors. The vector $\mathbf{r}$ is then

$$
\begin{aligned}
\mathbf{r} & =\left(\mathbf{U} \otimes \mathbf{I}_{N_{R}}\right) \mathbf{h}+\mathbf{z} \\
& =\left[\mathbf{r}_{1}^{T} \cdots \mathbf{r}_{N_{R}}^{T}\right]^{T} \\
\mathbf{z} & =\left[\mathbf{z}_{1}^{T} \cdots \mathbf{z}_{N_{R}}^{T}\right]^{T} \\
\mathbf{h} & =\left[\mathbf{h}_{1}^{T} \cdots \mathbf{h}_{N_{R}}^{T}\right]^{T},
\end{aligned}
$$

where the $\otimes$ operator represents the Kronecker product.

\section{B. Carrier Frequency Offset}

As presented in [5], the progressive phase rotations represented by the $\mathbf{D}$ matrix can be understood as a perturbation on the channel state that degrades the orthogonality of the transmit and receive filters. This provides motivation for finding a high accuracy CFO estimate.

The BS CFO matrix $\mathbf{D}_{b}\left(c_{b}\right)$ from (4) represents the rotation of the symbols due to the CFO of the $b^{\text {th }}$ BS LO, where

$$
\mathbf{D}_{b}\left(c_{b}\right)=\operatorname{diag}\left(1 e^{j c_{b}} \cdots e^{j c_{b}(N-1)}\right) .
$$

The variable $c_{b}=2 \pi f_{b} T_{s}$ represents the normalized CFO with a CFO of $f_{b}=\varepsilon_{0} f_{c} \mathrm{~Hz}$, a carrier frequency of $f_{c} \mathrm{~Hz}$, and a symbol period of $T_{s}$ seconds.

\section{CFO AND CSI ESTIMATION}

Training sequences for channel estimation are generally comprised of an initial long sequence, followed by periodic short sequences designed for estimate updates. Thus, the TSs necessarily take up a portion of the available channel throughput; the higher the required accuracy of the CFO and CSI estimates, the larger this ratio becomes. This loss in throughout can be partially mitigated by optimizing the TS length and symbol energy [8]. To further minimize the loss in throughput, TSs can be transmitted simultaneously from all BS antennas, a technique used in the joint $\mathrm{CFO} / \mathrm{CSI}$ estimators in [7] and [9]. However, these simultaneous training techniques were designed assuming a quasi-static channel. To begin with, we will provide a brief summary of some existing quasistatic channel CFO and CSI estimators for simultaneous TSs. The effects of the fading channel on these estimators will be covered in the following section.

For the quasi-static channel, all estimators referred to in this work rely upon a least-squares (LS) estimate of the channel state. The length- $B N N_{T}$ vector $\mathbf{h}$ from (7) is reduced to the length- $B N_{T}$ vector $\mathbf{h}_{Q}$ and the $N \times B N N_{T}$ matrix $\mathbf{U}(\mathbf{c})$ from (3) is reduced to the $N \times B N_{T}$ matrix $\mathbf{U}_{Q}(\mathbf{c})$ when the channel is quasi-static. Solving for the maximum likelihood (ML) joint CFO and CSI metric using (6) results in

$$
\Lambda_{h}\left(\mathbf{c}, \mathbf{h}_{Q}\right)=\left\|\mathbf{r}-\overline{\mathbf{U}}_{Q}(\mathbf{c}) \mathbf{h}_{Q}\right\|^{2},
$$


where $\overline{\mathbf{U}}_{Q}(\mathbf{c})=\mathbf{U}_{Q}(\mathbf{c}) \otimes \mathbf{I}_{N_{R}}$. The LS estimate $\hat{\mathbf{h}}_{Q}$ is used to remove the dependence of (9) upon $\mathbf{h}_{Q}$, where

$$
\hat{\mathbf{h}}_{Q}=\left(\overline{\mathbf{U}}_{Q}^{\dagger}(\mathbf{c}) \overline{\mathbf{U}}_{Q}(\mathbf{c})\right)^{-1} \overline{\mathbf{U}}_{Q}^{\dagger}(\mathbf{c}) \mathbf{r}
$$

Inserting (10) into (9) and simplifying results in the metric being solely dependent on $\mathbf{c}$,

$$
\Lambda_{Q}(\mathbf{c})=\sum_{m=1}^{N_{R}} \mathbf{r}_{m}^{\dagger} \mathbf{U}_{Q}(\mathbf{c})\left(\mathbf{U}_{Q}^{\dagger}(\mathbf{c}) \mathbf{U}_{Q}(\mathbf{c})\right)^{-1} \mathbf{U}_{Q}^{\dagger}(\mathbf{c}) \mathbf{r}_{m} .
$$

The estimate $\hat{\mathbf{c}}$ of $\mathbf{c}$ from maximization of (11) can then be used in (10) to find $\hat{\mathbf{h}}_{Q}$.

\section{A. Simultaneous TS}

In [9], Ahmed et al. exploit the fact that in certain cases, the off-diagonals of the covariance matrix $\mathbf{U}_{Q}^{\dagger}(\mathbf{c}) \mathbf{U}_{Q}(\mathbf{c})$ will be negligible compared to the diagonals. This approximation allows each CFO to be estimated independently. In practice, this approximation could be valid when the sequences are short enough to avoid accumulation of sufficient phase difference to alter the correlations between the TSs. We will refer to this technique as ST-AO for "simultaneous training - assumed orthogonalization." Since the performance of the ST-AO estimator is contingent on $\mathbf{U}_{Q}^{\dagger}(\mathbf{c}) \mathbf{U}_{Q}(\mathbf{c})$ being diagonal, it is very sensitive to accumulation of the CFO. As $N$ or the maximum CFO $c_{\max }$ increase and the off-diagonals of $\mathbf{U}_{Q}^{\dagger}(\mathbf{c}) \mathbf{U}_{Q}(\mathbf{c})$ grow, the sequences will be long enough to allow accumulation of the CFO. The result is an error floor at high SNR, which is dependent upon $N$ and the maximum normalized CFO $c_{\text {max }}$.

To compensate for the off-diagonals that occur in $\mathbf{U}_{Q}^{\dagger}(\mathbf{c}) \mathbf{U}_{Q}(\mathbf{c})$ without resorting to the computationally intensive full ML search, reduced complexity estimators based on the Newton method were proposed in [7]. These estimates were shown to converge to the Cramer-Rao Lower Bound at higher SNRs for all values of $c_{\max }$. We will refer specifically to the unsegmented ST-N technique, which stands for "simultaneous training - Newton."

The success of any simultaneous multiple CFO estimator based on the metric in (9) is contingent on the existence of the inverse of $\mathbf{U}_{Q}^{\dagger}(\mathbf{c}) \mathbf{U}_{Q}(\mathbf{c})$, and in turn $\mathbf{U}_{Q}(\mathbf{c})$ should satisfy $\operatorname{rank}\left(\mathbf{U}_{Q}(\mathbf{c})\right) \geq B N_{T}$. If this rank condition is not met, $\mathbf{U}_{Q}^{\dagger}(\mathbf{c}) \mathbf{U}_{Q}(\mathbf{c})$ is singular. Thankfully, the TS matrix $\mathbf{X}$ can be composed of orthogonal or quasi-orthogonal sequences. Doing this ensures that $\operatorname{rank}(\mathbf{X})=B N_{T}$, as advised in [10]. This in turn leads to $\operatorname{rank}\left(\mathbf{U}_{Q}(\mathbf{c})\right)=B N_{T}$.

\section{EFFEct of Time-VARYing Channel}

The following sections present results of the CFO and CSI estimators in fading channels using Monte Carlo simulations. All simulations use CFOs that are uniformly distributed between $\left[-c_{\max },+c_{\max }\right]$. The channels are Rayleigh distributed, with a mean SNR of $\Gamma_{B S}=E_{B S} / N_{0}$, and have a Jakes spectrum such that the time autocorrelation function for channel $h_{b, t, m}$ is

$$
E\left[h_{b, t, m}(n) h_{b, t, m}(p)\right]=J_{0}\left(2 \pi f_{D} T_{s}(n-p)\right) .
$$

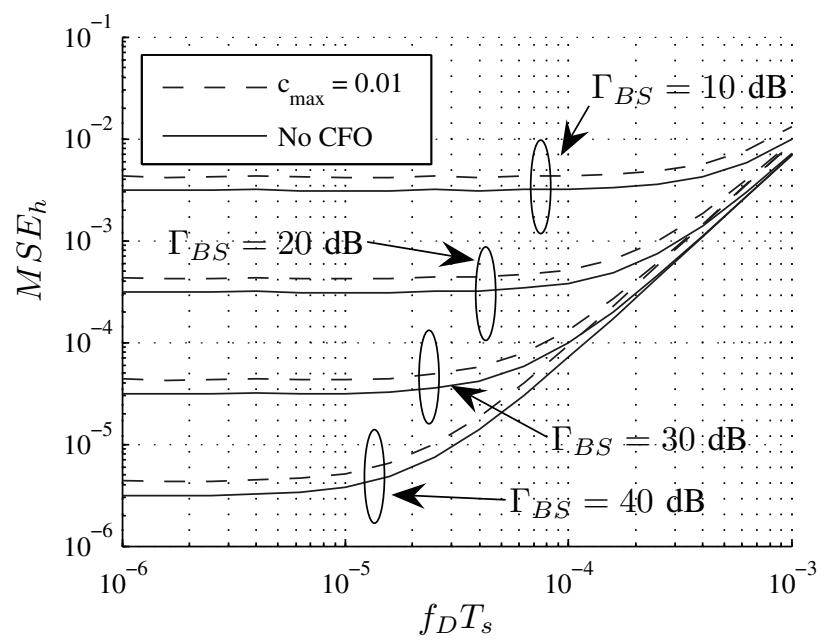

Fig. 2. Effect of Fading Channel on Sum CSI Estimate MSE of $\hat{\mathbf{h}}_{Q} ; B=4$, $N=64, N_{R}=N_{T}=2$

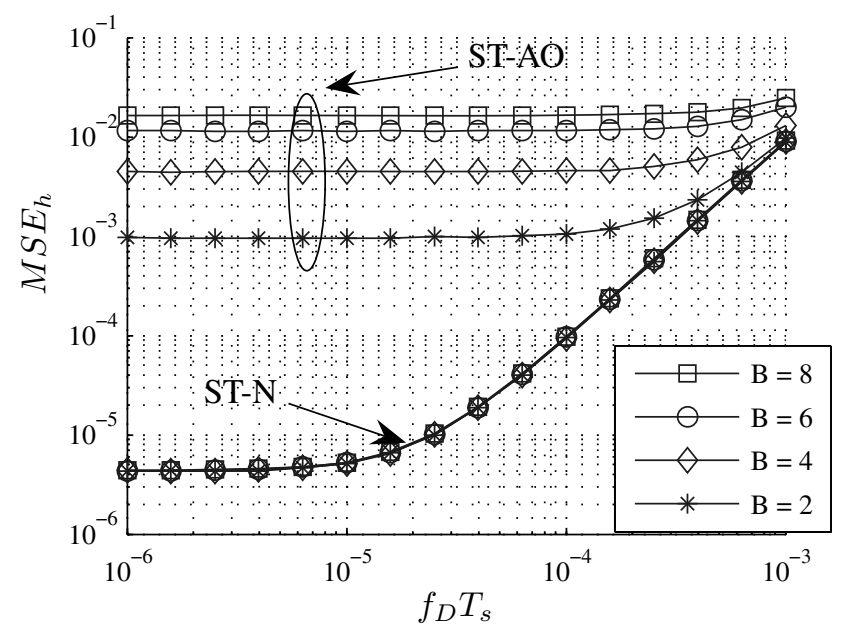

Fig. 3. Effect of Fading Channel on Sum CSI Estimate MSE of $\hat{\mathbf{h}}_{Q}$ for simultaneous TSs; $\Gamma_{B S}=40 \mathrm{~dB}, N=64, c_{\max }=0.01, N_{R}=N_{T}=2$

\section{A. CSI Estimate}

Since all of the estimators presented in Section III-A use a LS estimate of the CSI, the expression from (10) is used here. Fig. 2 demonstrates the effect of fast fading in a system with $B=4, N=64$, and the maximum CFO set to $c_{\max }=0$ and 0.01 . Since the actual CSI $\mathbf{h}$ changes from symbol to symbol and contains $B N N_{T}$ complex values, but the estimated CSI $\hat{\mathbf{h}}_{Q}$ is a snapshot and contains only $B N_{T}$ values, the mean square error (MSE) of the CSI is defined as

$$
M S E_{h}=\frac{1}{B N N_{T}} \sum_{n=1}^{N}\left|\mathbf{h}(n)-\hat{\mathbf{h}}_{Q}\right|^{2},
$$

where $\mathbf{h}(n)$ and $\mathbf{h}_{Q}$ are both length- $B N_{T}$ vectors. The figures show $M S E_{h}$ after averaging over $10^{4}$ Monte Carlo simulations. First, it is evident that non-zero CFO has minimal effect on the CSI MSE when compared to the SNR and fade rate. 


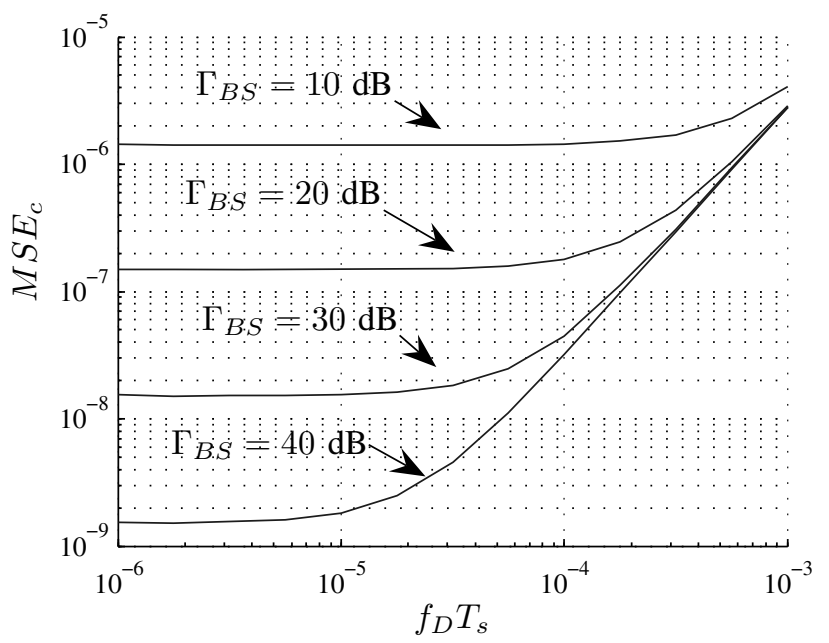

Fig. 4. Effect of Fading Channel on ST-N CFO Estimate MSE of $\hat{\mathbf{c}} ; B=4$, $N=64, c_{\max }=0.01, N_{R}=N_{T}=2$

Second, a threshold can be loosely defined above which an increase in the fade rate will result in a increase in $M S E_{h}$; this increase is proportional to the square of $f_{D} T_{s}$, due to the Jakes autocorrelation function being approximately quadratic for small argument values. This threshold can be used to determine a maximum frame size. Third, an increase in the SNR results in a linear drop in $M S E_{h}$, since there is no diversity effect in estimation of the CSI. Note that systems with higher SNR are more sensitive to the fading channel, and are subject to a lower fading threshold.

In Fig. 3, ST-AO and ST-N are compared over both the number of BSs and the fade rate. It is immediately obvious that ST-AO is incapable of handling the effect of the CFO, regardless of the fade rate. ST-AO cannot cope with additional BSs because it disregards any cross-correlation due to CFO on the symbols; additional BSs result in extra interference for the estimator. For ST-N, an increase in $B$ does not result in an increase in $M S E_{h}$, since ST-N accommodates the crosscorrelation coefficients in (11).

\section{B. CFO Estimate}

The effect of the fading channel on the ST-N CFO estimate follows the trend shown for the CSI estimate closely. Fig. 4 shows the results of the CFO MSE, defined as

$$
M S E_{c}=\frac{1}{B} \sum_{b=1}^{B}\left|c_{b}-\hat{c}_{b}\right|^{2},
$$

where an average of $10^{4}$ Monte Carlo simulations was used to generate the curves. Since the simultaneous TS CFO estimate relies inherently on the CSI estimate, it suffers the same degradation as the fade rate increases.

By using the diversity and extra SNR provided by multiple MS antennas, CFO estimator performance can be improved. Fig. 5 demonstrates this for a variety of $N_{R}$ values. For MS antennas that experience independent fading, additional antennas result in a downward shift of the MSE curve.

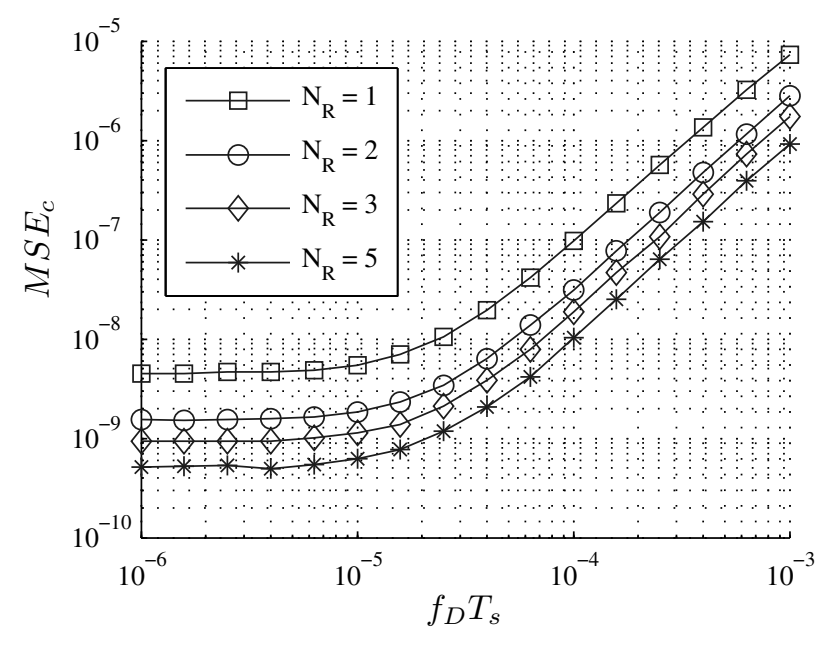

Fig. 5. Effect of Fading Channel on ST-N CFO Estimate MSE of $\hat{\mathbf{c}}$ for Diversity Antennas; $B=4, N=64, \Gamma_{B S}=40 \mathrm{~dB}, c_{\max }=0.01$, $N_{T}=2$

\section{Modification of the ML Metric}

The previous section showed that fast fading rapidly degrades the quality of multiple CFO estimates when the channel is assumed to be static over the duration of the training sequence. It is a new result, but somewhat to be expected. In this section, we show how to improve on the estimation by taking explicit account of the variability of the channel.

Given the assumption that the channel is time-varying, the $\mathbf{U}_{Q}(\mathbf{c})$ and $\mathbf{h}_{Q}$ in (9) must be replaced with $\mathbf{U}(\mathbf{c})$ and $\mathbf{h}$, as in (7). Examining $\mathbf{U}(\mathbf{c})$ and (9), the static channel estimate $\hat{\mathbf{h}}_{Q}$ becomes undefined; since the channel changes between each symbol time, the rank requirement of the TS matrix effectively changes to $\operatorname{rank}(\mathbf{U}(\mathbf{c}))=B N N_{T}$. This requirement is impossible to meet with a static channel estimate. A channel estimator capable of operating in the fading channel would be more appropriate. The channel estimator can then be integrated into the metric in (9), which can be rewritten as

$$
\Lambda_{h}(\mathbf{c}, \mathbf{h})=\|\mathbf{r}-\overline{\mathbf{U}}(\mathbf{c}) \mathbf{h}\|^{2} .
$$

To provide a good fading channel estimate, we suggest a parameter-based estimator that uses eigenvectors of the channel autocorrelation matrix as basis functions. This estimator is similar in construction to [11], which uses polynomials as basis functions. First, define the $N \times N$ Toeplitz channel autocorrelation matrix as $\mathbf{R}$, where $\mathbf{R}_{n, p}=J_{0}\left(2 \pi f_{D} T_{s}(n-p)\right)$. Next, the eigenvectors corresponding to the $P_{j}$ largest eigenvalues of $\mathbf{R}$ are represented in the $N \times P_{j}$ basis matrix $\mathbf{Q}_{j}$. The estimation parameters $\hat{\mathbf{a}}_{b, t, m}$ are then found such that

$$
\hat{\mathbf{h}}_{b, t, m}=\mathbf{Q}_{j} \hat{\mathbf{a}}_{b, t, m}
$$

is the channel estimate. A least squares solution to $\hat{\mathbf{a}}_{m}=$ $\left[\mathbf{a}_{1,1, m}^{T} \mathbf{a}_{1,2, m}^{T} \cdots \mathbf{a}_{B, N_{T}, m}^{T}\right]^{T}$ as a function of the CFO $\mathbf{c}$ is

$$
\hat{\mathbf{a}}_{m}(\mathbf{c})=\left(\mathbf{Q}^{\dagger} \mathbf{U}^{\dagger}(\mathbf{c}) \mathbf{U}(\mathbf{c}) \mathbf{Q}\right)^{-1} \mathbf{Q}^{\dagger} \mathbf{U}^{\dagger}(\mathbf{c}) \mathbf{r}_{m},
$$




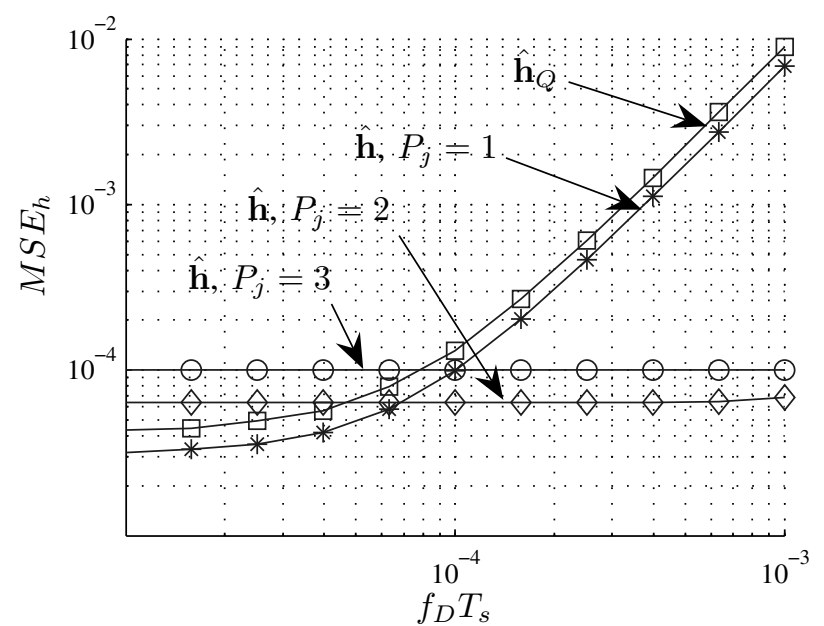

Fig. 6. Effect of Fading Channel on CSI Estimate MSE for $\hat{\mathbf{h}}_{Q}$ and $\hat{\mathbf{h}}$; $B=4, N=64, \Gamma_{B S}=30 \mathrm{~dB}, c_{\max }=0, N_{R}=N_{T}=2$

where $\mathbf{Q}=\mathbf{Q}_{j} \otimes \mathbf{I}_{B N_{T}}$. The expressions in (16) and (17) can be used to replace $\mathbf{h}$ in (15), resulting in a new ML metric

$$
\Lambda_{F}(\mathbf{c})=\sum_{m=1}^{N_{R}} \mathbf{r}_{m}^{\dagger} \mathbf{U}(\mathbf{c}) \mathbf{Q}\left(\mathbf{Q}^{\dagger} \mathbf{U}^{\dagger}(\mathbf{c}) \mathbf{U}(\mathbf{c}) \mathbf{Q}\right)^{-1} \mathbf{Q}^{\dagger} \mathbf{U}^{\dagger}(\mathbf{c}) \mathbf{r}_{m}
$$

Note that now the rank requirement changes to $\operatorname{rank}\left(\mathbf{Q}^{\dagger} \mathbf{U}^{\dagger}(\mathbf{c}) \mathbf{U}(\mathbf{c}) \mathbf{Q}\right) \geq B N_{T} P_{j}$, which is achievable as long as $N \geq B N_{T} P_{j}$ (since $\mathbf{Q}$ is $N \times B N_{T} P_{j}$ and $\left.\left.\operatorname{rank}(\mathbf{Q})=\min \left(N, B N_{T} P_{j}\right)\right)\right)$.

Fig. 6 compares the $M S E_{h}$ of $\hat{\mathbf{h}}$ in (16) to that of $\hat{\mathbf{h}}_{Q}$ in (10). The figure shows that the performance of $\hat{\mathbf{h}}$ is superior in fast fading. The gap between $\hat{\mathbf{h}}_{Q}$ and $\hat{\mathbf{h}}$ in slow fading is due to $P_{j}$. For every added parameter estimate, $M S E_{h}$ shifts upwards. However, the use of multiple parameter estimates reduces the CSI estimate sensitivity to $f_{D} T_{s}$. Combined with the results from Section IV-B, this suggests that the CFO estimate will have similar benefits when $P_{j}$ is chosen intelligently.

For the ST-N estimator in [7], this approach is not applicable. The reason is due to the non-convex surface of $\Lambda_{F}(\mathbf{c})$, which creates a number of local maxima. A sample $\Lambda_{F}(\mathbf{c})$ is shown in Fig. 7 for $B=2$ and $f_{D} T_{s}=10^{-3}$. The cause of the non-convexity is the non-linear basis matrix $\mathbf{Q}_{j}$. The use of $\Lambda_{F}(\mathbf{c})$ for CFO estimation will be the topic of a future study.

\section{Conclusions}

For synchronous cooperative multibase beamforming, once the CFO is accurately removed, channel instability remains as the sole limiting factor. The results demonstrate that above a certain fade rate threshold, the MSE of both the CFO and CSI estimates from existing schemes degrade on the order of the square of the normalized fade rate. To accommodate for the fading channel, modifications were made to the joint CFO/CSI ML metric. The modifications used a parameterized fading channel estimate based on the eigenvectors of the

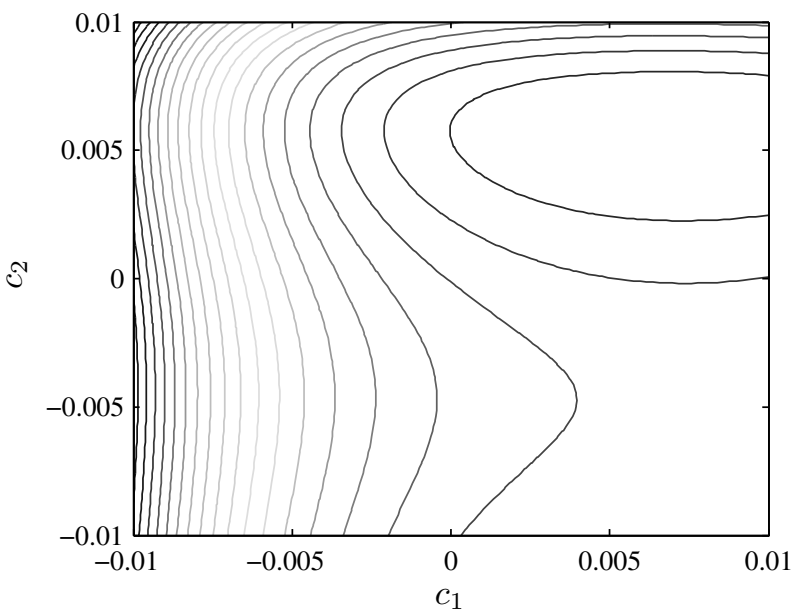

Fig. 7. Sample $\Lambda_{F}(\mathbf{c}) ; f_{D} T_{s}=10^{-3}, B=2, N=64, \Gamma_{B S}=30 \mathrm{~dB}$, $c_{\max }=0.01, N_{R}=N_{T}=2$

channel autocorrelation matrix. It was shown that the MSE of the new CSI estimate is insensitive to increases in the fade rate. For the example of $f_{D} T_{s}=10^{-3}$ and $B=4$, the parameterized fading channel estimate maintains a MSE value of $10^{-4}$ while the linear estimate climbs to a MSE value of $10^{-2}$. An investigation into CFO estimates using the new metric is forthcoming.

\section{REFERENCES}

[1] S. J. Grant and J. K. Cavers, "Performance enhancement through joint detection of cochannel signals using diversity arrays," IEEE Trans. Commun., vol. 46, no. 8, pp. 1038-1049, 1998.

[2] S. V. Hanly, "Capacity and power control in spread spectrum macrodiversity radio networks," IEEE Trans. Commun., vol. 44, no. 2, pp. 247-256, 1996.

[3] L. Welburn, J. K. Cavers, and K. W. Sowerby, "Multiuser-macrodiversity detection in rayleigh fading channels," in IEEE Veh. Tech. Conf., vol. 5, 2000, pp. 2340-2347.

[4] M. N. Bacha, J. S. Evans, and S. V. Hanly, "On the capacity of mimo cellular networks with macrodiversity," in IEEE Australian Commun. Theory Workshop, vol. 1, 2006, pp. 105-109.

[5] B. W. Zarikoff and J. K. Cavers, "Carrier frequency offset in coordinated multibase mimo narrowband systems," in IEEE 67th Veh. Technol. Conf., to be published.

[6] O. Besson and P. Stoica, "On parameter estimation of mimo flat-fading channels with frequency offsets," IEEE Trans. Signal Process., vol. 51, no. 3, pp. 602-613, 2003.

[7] B. W. Zarikoff and J. K. Cavers, "Multiple frequency offset estimation for the downlink of coordinated mimo systems," IEEE J. on Select Areas Commun., to be published.

[8] B. Hassibi and B. M. Hochwald, "How much training is needed in multiple-antenna wireless links?" IEEE Trans. Inform. Theory, vol. 49, no. 4, pp. 951-963, 2003.

[9] S. Ahmed, S. Lambotharan, A. Jakobsson, and J. A. Chambers, "Mimo frequency-selective channels with multiple-frequency offsets: estimation and detection techniques," IEE Proc. Commun., vol. 152, no. 4, pp. 489494, 2005.

[10] P. Stoica and O. Besson, "Training sequence design for frequency offset and frequency-selective channel estimation," IEEE Trans. Commun., vol. 51, no. 11, pp. 1910-1917, 2003.

[11] W. S. Leon and D. P. Taylor, "Generalized polynomial-based receiver for the flat fading channel," IEEE Trans. Commun., vol. 51, no. 6, pp. 896-899, 2003. 